\title{
Derecho Disciplinario en Colombia, desde la imposición de Sanciones ¿la pérdida de su vocación preventiva?i
}

\author{
Disciplinary law in Colombia from the imposition of sanctions, is it the loss of \\ its preventive vocation?
}

\section{Por: Ivonne Pamela Hernández Villamizar, ${ }^{1}$ Julián David Guachetá Torres, ${ }^{2}$ Hoover Hugo Paredes Mosquera ${ }^{3}$ \& Esperanza del Carmen Reyes Gómez ${ }^{4}$}

1. Doctora en Derecho de la Universidad de Salamanca, magíster en Corrupción y Estado de Derecho del mismo claustro, especialista en Contratación Estatal y su Gestión del Colegio Mayor Nuestra Señora del Rosario, con estudios de postdoctorado en la Universidad de Messina (Italia). Docente investigadora de la Universidad Militar Nueva Granada. Contacto: ivonne.hernandez@unimilitar.edu.co Orcid: https://orcid.org/0000-0001-5772-3021 Scholar: https://scholar.google.com/ citations?hl=es\&user=|w-jE9EAAAAJ

2. Abogado de la Corporación Universitaria Autónoma del Cauca y politólogo de la Universidad del Cauca, actualmente se encuentra vinculado como Joven Investigador de la Facultad de Derecho de la Corporación Universitaria Autónoma del Cauca. Integrante del Grupo de Investigación de Ciencias Sociales y Humanas. Colombia. Contacto: julian.guacheta.t@uniautonoma.edu.co Orcid: https://orcid.org/0000-0003-1697-9463 Scholar: https://scholar.google.com/ citations? user=0EycsnOAAAAJ\&hl=en

3. Abogado Especialista y Magister en Derecho Administrativo de la Universidad del Cauca, estudiante del Doctorado en Derecho Administrativo de la Universidad Libre sede Bogotá, integrante del Grupo de Investigación de Ciencias Sociales y Humanas, categoría C. Actualmente es Vicerrector de Investigación en la Corporación Universitaria Autónoma del Cauca, Colombia. Contacto: hoover.paredes.m@uniautonoma.edu.co Orcid: https://orcid.org/0000-0002-9845-884X Scholar: https:// scholar.google.es/citations?user=rcvZhW8AAAAJ\&hl=es

4. Administradora de Empresas, Abogada, Especialista en Derecho Penal, Directora Consultorio Jurídico y docente de la Corporación Universitaria Autónoma del Cauca. Colombia. Contacto: esperanza.reyes.g@uniautonoma.edu.co Orcid: https://orcid.org/0000-0002-3836-3590

\section{OPEN ACCESS}

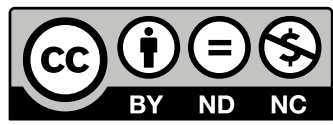

Copyright: ( $\odot 2020$ El Ágora USB.

La Revista El Ágora USB proporciona acceso abierto a todos sus contenidos bajo los términos de la licencia creative commons AtribuciónNoComercial-SinDerivar 4.0 Internacional (CC BY-NC-ND 4.0)

Tipo de artículo: Investigación

Recibido: julio de 2019

Revisado: agosto de 2019

Aceptado: octubre de 2019

Doi: 10.21500/16578031.4204

Citar así: Hernández Villamizar, I., Guachetá Torres, J., Paredes Mosquera, H. y Reyez Gómez, E. (2020). Derecho Disciplinario en Colombia, desde la imposición de sanciones ¿la pérdida de su vocación preventiva? El Ágora USB, 20(1). 66-81.

Doi: $10.21500 / 16578031.4204$

\section{Resumen}

El aumento progresivo de las sanciones disciplinarias no es un hecho aislado o ajeno a las dinámicas sociales y políticas de Colombia, podemos decir, que tanto el desarrollo normativo en términos de creación de leyes así como las diferentes posturas jurisprudenciales respecto al tema influyen en la tendencia de crecimiento; también lo hacen las condiciones sociales y los eventos coyunturales que involucran responsabilidad de funcionarios del Estado y, específicamente, en el aumento de funcionarios públicos, se puede ver la relación entre la concentración de funcionarios en un territorio y las sanciones disciplinarias que se imponen en este.

Palabras clave. Derecho público; derecho disciplinario; funcionario público; normatividad; sanción disciplinaria.

\section{Abstract}

The progressive increase in disciplinary sanctions is not an isolated or unrelated fact to the social and political dynamics of Colombia. It can be said that both regulatory development in terms of the creation of laws, as well as the different jurisprudential postures on the issue influence the growth trend, so do social conditions and contemporary events, which involve responsibility for State officials. Moreover, specifically, in the increase of public officials, the relationship between the concentration of civil servants in a territory and the disciplinary sanctions, which are imposed in it, can be appreciated.

Keyword. Public Law; Disciplinary Law; Regulation; and Disciplinary Sanction. 


\section{Introducción}

Esta investigación se desarrolló en torno a la pregunta ¿Cuál ha sido el desarrollo histórico del derecho disciplinario y su aplicación en Colombia desde el año 2000 hasta el 2018?, para dar respuesta a esta interrogante fue necesario definir tres aspectos sustanciales. El primero, fue la identificación de los cambios normativos del Derecho Disciplinario en Colombia; el segundo, la descripción de la normatividad actual; y, el tercero, determinar la aplicación de la norma disciplinaria en Colombia, teniendo en cuenta investigaciones y sanciones. De esta forma, se logró establecer la evolución normativa del derecho disciplinario y su aplicación en Colombia desde el año 2000 hasta 2018.

De acuerdo a lo anterior, nuestra hipótesis de trabajo indicó que, el número de investigaciones y sanciones disciplinarias a funcionarios del Estado, han aumentado debido a la pérdida de la vocación preventiva y organizacional del derecho disciplinario, que ha sido reemplazada por una vocación punitiva, conforme se han llevado a cabo las reformas normativas en esta rama del derecho. Esto quiere decir que, a mayores reformas normativas, es más elevado el número de procesos disciplinarios; en razón a que las modificaciones han restringido en mayor medida el comportamiento de los funcionarios del Estado, generando como consecuencia un aumento de investigaciones y sanciones disciplinarias.

\section{Metodología}

Se acudió al método hipotético deductivo, lo cual significó el planteamiento de la hipótesis anterior, que a partir de la evidencia y de los resultados encontrados pudo ser corroborada. En ese sentido, se utilizaron tres técnicas de investigación:

La primera técnica es el análisis documental, tanto de producción académica o doctrina, así como, de la normatividad que compone el marco jurídico del derecho disciplinario en Colombia y, por último, de la jurisprudencia y precedentes emanados de órganos, tribunales o entidades encargadas de adoptar medidas disciplinarias.

La segunda técnica es la estadística, frente al tratamiento y presentación de los datos de las entidades encargadas del control disciplinario en Colombia, y

La tercera técnica, la entrevista, para conocer opiniones centrales de académicos o funcionarios del Estado que tienen relación directa con el Derecho Disciplinario en Colombia.

\section{Resultados}

\section{Desarrollo Normativo del Derecho Disciplinario en Colombia}

Es innegable el rol protagónico que tiene el derecho disciplinario para la realidad política colombiana, las investigaciones y posteriores sanciones son analizadas y seguidas de cerca por amplias coberturas mediáticas, y las facultades legales de los máximos órganos de control en materia disciplinaria se enfrentan con los demás poderes públicos del Estado. Pero esta situación no siempre ha sido así, "La última década del siglo xx y lo que va corrido del XXI pueden calificarse, en Colombia como la era del estudio científico del Derecho disciplinario" (Gómez Pavajeau, 2011). Este hecho, solo ha sido posible por medio de la autonomía que el derecho disciplinario ha logrado respecto a otras áreas de mayor tradición, como lo son el derecho Penal o el derecho Administrativo. Sin embargo, antes de analizar el proceso mediante el cual el Derecho Disciplinario ha logrado su autonomía, y establecer el desarrollo normativo que esto significó, es necesario plantear algunas aproximaciones teóricas y prácticas que definen al derecho disciplinario.

En primer lugar, debemos hacer mención al nivel constitucional del derecho disciplinario, ya que de este, se va a desprender todo el desarrollo legal y jurisprudencial del tema en cuestión. La primera mención respecto a la responsabilidad de los servidores del Estado la encontramos en el título primero de la Constitución Política, de los Principios 
Fundamentales, en donde el artículo sexto de la Carta establece que: "Los particulares sólo son responsables ante las autoridades por infringir la Constitución y las leyes. Los servidores públicos lo son por la misma causa y por omisión o extralimitación en el ejercicio de sus funciones" (Constitución Política de Colombia, 1991); esta disposición, como se puede apreciar, le asigna una responsabilidad adicional a los servidores públicos, quienes no solo se deben preocupar por no infringir la Constitución y las leyes, sino también por el mal ejercicio de su cargo, en el sentido de ir más allá de sus funciones o dejar de realizar las obligaciones que la ley les impuso. Es precisamente a partir de esta norma, en donde se hace menester un cuerpo normativo que permita establecer la responsabilidad de los funcionarios en los eventos señalados.

En ese orden de ideas, la Constitución, en el capítulo dos sobre la Organización del Estado, indica en su artículo 124 que "La ley determinará la responsabilidad de los servidores públicos y la manera de hacerla efectiva" (Constitución Política de Colombia, 1991). Esto implicó que la promulgación de una normatividad que regule y describa los procesos en donde se demostrase la responsabilidad de los servidores públicos, significara una obligación constitucional, por esta razón se puede afirmar que, el derecho disciplinario es una necesidad y una herramienta para la definición de las relaciones especiales de sujeción de los funcionarios públicos con el Estado colombiano y la responsabilidad derivada de sus actuaciones (Isaza Serrano, 2009).

La relación especial de sujeción incluye aspectos relacionados con funciones y fines del derecho disciplinario, que deben soportar los servidores públicos en aras del interés general y el bienestar común; los cuales se intensifican en los estatutos disciplinarios de la Policía Nacional y las Fuerzas Militares; existiendo entonces unas relaciones especiales de sujeción moderadas y otras especializadas (Gómez Pavajeu, 2009).

Ha sido la misma Constitución la que ha extendido la responsabilidad disciplinaria, indicando en su artículo 123 que, cuando se hace referencia a los servidores públicos se incluyen a los miembros de las corporaciones públicas, los empleados y trabajadores del Estado y de sus entidades descentralizadas territorialmente y por servicios.

La Ley 1952 de 2019 establece en el Libro III del Régimen Especial, en su Título I sobre Régimen de Los Particulares, en el artículo 70 que "el presente régimen se aplica a los particulares que ejerzan funciones públicas de manera permanente o transitoria; que administren recursos públicos [...]" (Ley 1952, 2019).

Luego menciona que los servidores públicos están al servicio del Estado y de la comunidad, por lo que deberán ejercer sus funciones en la forma prevista por la Constitución, la ley y el reglamento (Constitución Política de Colombia, 1991). Lo anterior remite nuevamente a la necesidad de un cuerpo normativo que describa las situaciones de las cuales se desprende la responsabilidad disciplinaria, así como de las sanciones correspondientes y las características de los procesos ante los organismos de control.

El desarrollo jurisprudencial, por otra parte, ha sido fundamental para lograr la autonomía del derecho disciplinario en Colombia. Inicialmente, respecto a su definición, en la Sentencia C-417 de 1993, la Corte Constitucional plantea una aproximación conceptual reuniendo varios elementos indispensables del derecho disciplinario, indicando que:

El derecho disciplinario está integrado por todas aquellas normas mediante las cuales se exige a los servidores públicos un determinado comportamiento en el ejercicio de sus funciones, independientemente de cuál sea el órgano o la rama a la que pertenezcan. (Sentencia C-417, 1993).

A partir de este pronunciamiento podemos distinguir, en primer lugar, que el derecho disciplinario no se concentra en una norma en específico o en un principio constitucional en concreto, sino que se configura como un marco normativo autónomo que debe estar 
acorde a los principios constitucionales. Posteriormente, la Corte Constitucional reiteró que el derecho disciplinario "es consustancial a la organización política y absolutamente necesario en un Estado de derecho" (Sentencia C-280, 1996), entendiendo la necesidad de un cuerpo normativo autónomo y completo en términos de determinar los deberes, prohibiciones, régimen de inhabilidades e incompatibilidades, así como las normas de procedimiento necesarias. De esta forma, se podría asegurar que la función pública sea ejercida en beneficio de los ciudadanos, y para garantizar la protección de los derechos y libertades; así como los derechos y funciones de los servidores públicos, por medio de la determinación de las faltas, procedimientos y sanciones aplicables a estos servidores (Instituto de Estudios del Ministerio Público, 2007).

\section{Principales reformas}

Si bien es cierto, solo a partir de 1995 se puede hablar de unificación o consolidación en un mismo cuerpo normativo de las regulaciones en materia disciplinaria en Colombia, existen antecedentes sobre los cuales resulta necesario referirse, en atención al objetivo de este estudio, en el desarrollo histórico del derecho disciplinario. Lo primero será remontarnos a la Ley 4 de 1913, sobre el Régimen político y municipal que, en defensa de los intereses de Estado, en todos sus niveles, y por tanto de la sociedad, delimitó el accionar del Ministerio Público, y estableció la competencia de su ejercicio en la Cámara de Representantes y el Procurador General de la Nación, los Fiscales de los que trataba el Código judicial de la época y los Personeros Municipales y empleados especiales.

Fue mediante este el instrumento que se atribuyó al Procurador General de la Nación las funciones de cuidar que todos los servidores públicos al servicio de la Nación desempeñaran cumplidamente sus deberes, acusar ante la Corte Suprema a los funcionarios cuyo juzgamiento correspondiera a esta corporación, cuidar que los demás funcionarios del Ministerio Público desempeñaran fielmente su cargo, y a promover que se les exigiera la responsabilidad por las faltas que cometieran, a dar las instrucciones que estimara convenientes a los empleados del ramo, para el mejoramiento en el desempeño de las funciones de estos, a defender los bienes e intereses del Estado y vigilar que sean administrados con celo e interés; todas ellas directamente relacionadas con la salvaguarda de la función pública (Ley 4, 1913).

Veinticinco años más tarde, fue promulgada la Ley 165 de 1938, por la cual se creó la carrera administrativa, el artículo 8 contempló los deberes de los empleados que a esta se vincularan, otorgando relevancia a la lealtad al espíritu de la Constitución, la eficiencia, imparcialidad y discreción de los servidores, al cumplimiento de sus funciones y sujeción a las órdenes superiores (Ley 165, 1938); también en ella, se identifica la primera referencia que vincula la honorabilidad y la buena fama en el comportamiento social del servidor, propios de su esfera privada, como un requisito para el ejercicio de la función pública, so pena de la pérdida de las prerrogativas de la carrera administrativa.

Enseguida, en 1939 mediante el Decreto Ley 2091, a través del cual se reglamentó la carrera administrativa, se establecieron las penas disciplinarias y el procedimiento para la aplicación de sanciones en este ámbito. Son penas disciplinarias: la amonestación, la multa hasta el monto de un sueldo mensual, el traslado a otro puesto y la remoción (Ley $734,2002)$.

Su artículo 18 reiteró lo dispuesto en la Ley 165 de 1938, frente a los deberes de los empleados de la Carrera, tomándose esta vez el legislador el trabajo de determinar cuándo existía falta de honorabilidad, y en qué consistía la buena fama, lo que sin duda a falta de tal especificidad dificultaría la aplicación de la norma disciplinaria, tal vez la misma problemática que hoy se afronta, al encontrar tipos referentes al 'buen comportamiento' del servidor público, sin una clara delimitación de lo correcto o lo erróneo, cuando lo que se juzga no genera una afectación directa la función. 
De igual manera, y hasta 1995, el legislador se ocupó de manera dispersa del control disciplinario, incluyendo referencias en cada una de las normas que regulaban la carrera administrativa, entre ellas el Decreto 1192 del 11 de julio de 1940, el Decreto 1679 de 1960, los Decretos Leyes 2400 y 3074 de 1968. Más adelante, la Ley 20 de 1972 referente a la composición y funcionamiento del Tribunal Disciplinario, y en 1973 con la reglamentación de los Decretos Ley 2400 y 3074 de 1968, mediante el Decreto 1950 de 1973, el que clasificó las faltas en graves y leves, así como la forma de graduar las sanciones.

Con mayor énfasis en el control disciplinario, se expidió la Ley 13 de 1984, reglamentada por el Decreto Nacional 482 de 1985 y modificada por la Ley 27 de 1992, en ella se estableció como objetivo del régimen disciplinario el aseguramiento de la sociedad, y de la administración pública, a la eficiencia en la prestación de los servicios a cargo del Estado, y su pertenencia al sistema de administración de personal; trayendo de presente además, la exigencia de moralidad, responsabilidad y conducta correcta de los funcionarios públicos. El legislador se ocupó también, en esta oportunidad, de establecer los derechos de los investigados, la conceptualización de falta disciplinaria, la calificación de las faltas, y la clase de sanciones.

Posteriormente, en 1995 es expedido el Estatuto Anticorrupción, Ley 190, la antesala de la normativa en la que centraremos los siguientes párrafos, dictado para preservar la moralidad en la Administración Pública y fijar las disposiciones para erradicar la corrupción administrativa. En este punto es menester aclarar que, se abordará de manera detallada la normativa expedida con posterioridad a la Constitución Nacional de 1991, por cuanto a través de ella se adoptó un modelo de Estado completamente distinto al que venía manejándose según la Constitución de 1886. Así las cosas, lo primero será referirse a la Ley 200 de 1995, del 28 de julio, por la cual se adoptó el Código Disciplinario Único, para luego abordar la Ley 734 de 2002, vigente por diecisiete años, y a la luz de la cual se fallaron la mayoría de procesos objeto de este estudio, y que fue modificada apenas hace unos meses por el Código General Disciplinario Ley 1952 de 2019.

Dicho lo anterior, y volviendo al argumento principal de estas líneas, surge un cuestionamiento que, a simple vista, va en contravía de lo afirmado en relación con el nuevo modelo de Estado: ¿Por qué si la Constitución de 1991 nos enmarca en un Estado Social de Derecho, en el que los derechos fundamentales y su garantía adquieren el mayor de los alcances, se ha incrementado la rigurosidad de la potestad disciplinaria del Estado?, pese a ello, en realidad, puede llevarnos a concluir que efectivamente el legislador ha excedido su autonomía, y ha incluido dentro del catálogo faltas y conductas que distan de afectar la función pública.

En efecto, lo que para algunos, entre ellos el ex procurador general de la nación Alejandro Ordoñez, constituyó una de las debilidades del primer Código Disciplinario Único, por cuanto la relación de faltas fue escasa y taxativa (Instituto de Estudios del Ministerio Público, 2011), puede percibirse de manera distinta al evidenciar, como se pretende, que dichas conductas y faltas son suficientes, cuando el objetivo de la aplicación del control disciplinario se encamina únicamente hacia la preservación de la función pública.

Considerando que la Ley 200 de 1995 calificaba como faltas gravísimas diez conductas, relacionadas con la obstaculización y negligencia en la investigación disciplinaria, administrativa o judicial, con el indebido incremento o provecho patrimonial derivado del ejercicio de sus funciones, aquellas referentes a la comisión de los delitos de genocidio o sometimiento de un grupo por razones étnicas, sociales y religiosas (Sentencia C-181, 2002), y aquellos relacionados con la privación de la libertad de una persona tendiente a su desaparición. También, las conductas orientadas a actividades políticas de respaldo y financiación con recursos del Estado, y por último contenidas en los numerales 7, 8, 9 y 10, asociadas directamente con el servicio, dentro de las que se encontraban el abandono 
injustificado del cargo (Corte Constitucional de Colombia, 1998), la publicación o utilización indebida de secretos oficiales (Corte Constitucional de Colombia, 1996), y el actuar a sabiendas de estar incurso en causales de incompatibilidad, inhabilidad, impedimento o conflicto de intereses, establecidos en la Constitución o en la Ley (Sentencia C-181, 2002).

Las mencionadas conductas que, a la luz de artículo 27 de la misma Ley, podían ser clasificadas como graves o gravísimas conforme a los criterios: grado de culpabilidad, de perturbación del servicio, naturaleza esencial del servicio, la falta de consideración para con los administrativos, la reiteración de la conducta, la jerarquía y mando del servidor público, la naturaleza y efectos de la falta, las modalidades y circunstancias del hecho, y los motivos determinantes. Es decir, el abanico de conductas objeto de sanción disciplinaria era reducido, y todas ellas afectaban directamente el servicio.

No obstante, las falencias percibidas en el primer Código disciplinario, dieron lugar a la expedición de la Ley que tuviera vigencia por diecisiete años, una herramienta llamada a dar solución a aspectos considerados negativos en su antecedente, los que pueden ser simplificados en cuatro grupos, el primero relacionado con el régimen de sanciones, que impedía la imposición de corrijas acordes con la gravedad de las conductas debido a la enumeración escasa y taxativa de las faltas gravísimas; la ausencia de un verdadero régimen especial para los particulares que ejercieran funciones públicas, debido a la no regulación de los deberes y prohibiciones que les fueran propios, así como de la sanciones; y los dos últimos, en materia de derechos humanos, dado que urgía adecuar la ley disciplinaria a las decisiones de la Corte Constitucional en este campo, y alcanzar la tipificación como faltas disciplinarias de las graves violaciones de los derechos humanos (Bernal Cuéllar, 1999).

Como resultado, la Ley 734 de 2002, al amparo de la cual se libraron los fallos analizados en esta investigación. Una apuesta por la independencia de esta rama del saber, a través de la cual el operador disciplinario logró entre otros, desligar sus decisiones del derecho penal y administrativo, para así unificar los criterios en la aplicación del derecho (Instituto de Estudios del Ministerio Público, 2011). Con ella además, el legislador estableció como principios rectores la función propia de la sanción administrativa disciplinaria, que es de naturaleza preventiva y correctiva, y mediante la cual se garantiza la efectividad de los principios y fines constitucionales y legales que deben observarse al ejercer la función pública, y el principio de proporcionalidad estableciendo expresamente la sanción que correspondía a cada clase de falta, según ésta sea gravísima, grave o leve y se cometa con dolo o culpa, también implantó el carácter garantista de la ley disciplinaria al incluir el derecho a la defensa material y a la designación de un abogado (Bernal Cuéllar, 1999).

En cuanto al procedimiento, a través del principio rector consagrado en el artículo 12 de la Ley 734 de 2002, se dotó de celeridad a la actuación disciplinaria, que se desarrolló en el cuerpo de la norma previendo los términos adecuados para cada una de la actuaciones, cuya fijación tuvo en cuenta factores como el número de investigados y la gravedad de la conducta; este último, cuando la misma tuviera relación con graves violaciones a los derechos humanos, un ámbito en el cual el operador disciplinario tuvo que irrumpir dado el contexto del conflicto armado interno en Colombia, y en estrecha relación la permisión a las organizaciones no gubernamentales de derechos humanos de intervención en los procesos disciplinarios y la revocatoria directa de la que trató el parágrafo $1^{\circ}$, del artículo 122, cuando se tratara de faltas disciplinarias que constituyeran violaciones al Derecho Internacional de los Derechos Humanos y del Derecho Internacional Humanitario, dando incluso oportunidad a las víctimas de su solicitud.

Como se indicó, el Código Disciplinario Único de 2002, estuvo vigente por diecisiete años, un lapso que da cuenta de cierta estabilidad en la materia, y se mantuvo hasta este lustro, aunque la iniciativa de su reforma data del año 2014, con la presentación del Proyecto 
de Ley No. 55 de 2014, autoría de los entonces Defensor del Pueblo y Procurador General de la Nación, orientado a la expedición de un Código de disciplina más claro, eficiente, práctico, proporcionado y, muy especialmente, aumentado en gran medida las garantías y los derechos fundamentales de quien debe ser investigado (Congreso de la República de Colombia, 2014). El proyecto fue materializado en la Ley 1952 del 28 de enero de 2019, por medio de la cual se expide el Código General Disciplinario, se derogan la Ley 734 de 2002 y algunas disposiciones de la Ley 1474 de 2011, relacionadas con el derecho disciplinario, sobre la cual no haremos especial referencia, al exceder ella el límite temporal de este estudio.

Teniendo en cuenta lo anterior, se debe hacer mención a los organismos de control en materia disciplinaria, siendo importante destacar que la función disciplinaria no descansa en un solo ente u organismo, pues si bien existe una cláusula general de competencia, en cabeza de la Procuraduría General de la Nación, la cual aplica a quienes se encuentren en ejercicio de funciones públicas, además de los miembros de las corporaciones de elección popular, es decir, que goza de una prerrogativa del poder disciplinario preferente, hay salvedades cuando el servidor público goza de un fuero especial, es el caso de los miembros de la fuerza pública Ejército Nacional y Policía Nacional. Se trata de normas disciplinarias especiales que se aplican a un número específico de funcionarios públicos, teniendo en cuenta las actividades que desarrollan, generalmente son regímenes más estrictos, un ejemplo de esto es el régimen especial de la fuerza pública (Instituto de Estudios del Ministerio Público, 2009).

\section{Aplicación de la norma disciplinaria en Colombia desde investigaciones}

hasta sanciones desde 2000 hasta 2018

Para dar cuenta de la aplicación de la norma disciplinaria en Colombia es necesario conocer la naturaleza jurídica de esta rama del derecho, algunos elementos de la razón ontológica se pueden encontrar en el artículo quinto del Código General Disciplinario, el cual establece que: "La sanción disciplinaria tiene finalidad preventiva y correctiva [...]" (Ley 1952, 2019), así mismo el artículo 11 indica que "Las finalidades del proceso son la prevalencia de la justicia, la efectividad del derecho sustantivo, la búsqueda de la verdad material y el cumplimiento de los derechos y garantías [...]" (Ley 1952, 2019). Por lo anterior, se puede inferir que el derecho disciplinario no tiene una función punitiva, sino que su naturaleza es de carácter preventiva y organizacional, para lo cual utiliza la imposición de una sanción disciplinaria como una herramienta para cumplir dichos objetivos, esto implica que, la sanción no debe ser el eje fundamental o el fin último, sino que debe ser considerada como un instrumento jurídico para orientar la conducta de los servidores públicos al cumplimiento de los fines del Estado (Mora, 2009).

Nuestro argumento central se relaciona con el llamado a retomar la vocación preventiva y organizativa del derecho disciplinario, la que se ha diluido en un derecho disciplinario punitivo, orientado a la aplicación de sanciones y la imposición de restricciones cada vez más amplias a los comportamientos de los funcionarios públicos, en donde la corrección del funcionario y el mejoramiento del actuar administrativo, son meros pretextos para una represión política (Nieto, 2003). Esta pérdida de vocación se ve reflejada en el aumento progresivo del número de sanciones que año a año se vienen imponiendo por las diferentes entidades competentes para realizarlo, por lo que es válido preguntarse si ¿más sanciones implican mayor efectividad de la norma disciplinaria? o por el contrario, son una muestra del inadecuado desarrollo de la vocación preventiva.

Teniendo en cuenta el artículo segundo de la Ley 1952 de 2019, o Código General Disciplinario, la titularidad de la potestad disciplinaria y autonomía de la acción está a cargo del Estado, por medio del poder preferente de la Procuraduría General de la Nación y de 
las Personerías Distritales y Municipales, las oficinas de control disciplinario interno, los funcionarios con potestad disciplinaria de las ramas, órganos y entidades del Estado (Ley 1952, 2019).

Debemos ser cuidadosos, pues si bien el aumento de las sanciones es notorio, esto puede responder a varias razones que a continuación se analizan con detenimiento, partiendo de la premisa de que el aumento de las sanciones disciplinarias es un hecho jurídico que requiere atención.

Antes de presentar la gráfica sobre el aumento de las sanciones disciplinarias, es menester indicar que desafortunadamente se cuenta con información limitada, pues los datos obtenidos solo abarcan desde el año 2008 hasta el 2017, información proporcionada por la Procuraduría General de la Nación, a través de la oficina de Sistema de Información de Registro de Sanciones y Causas de Inhabilidad, en adelante SIRI, y recogida por el Observatorio de Transparencia y Anticorrupción, por medio de su Indicador de sanciones disciplinarias. Pese a lo anterior, esta información nos permite evidenciar tendencias y datos globales que soporten los argumentos expuestos.

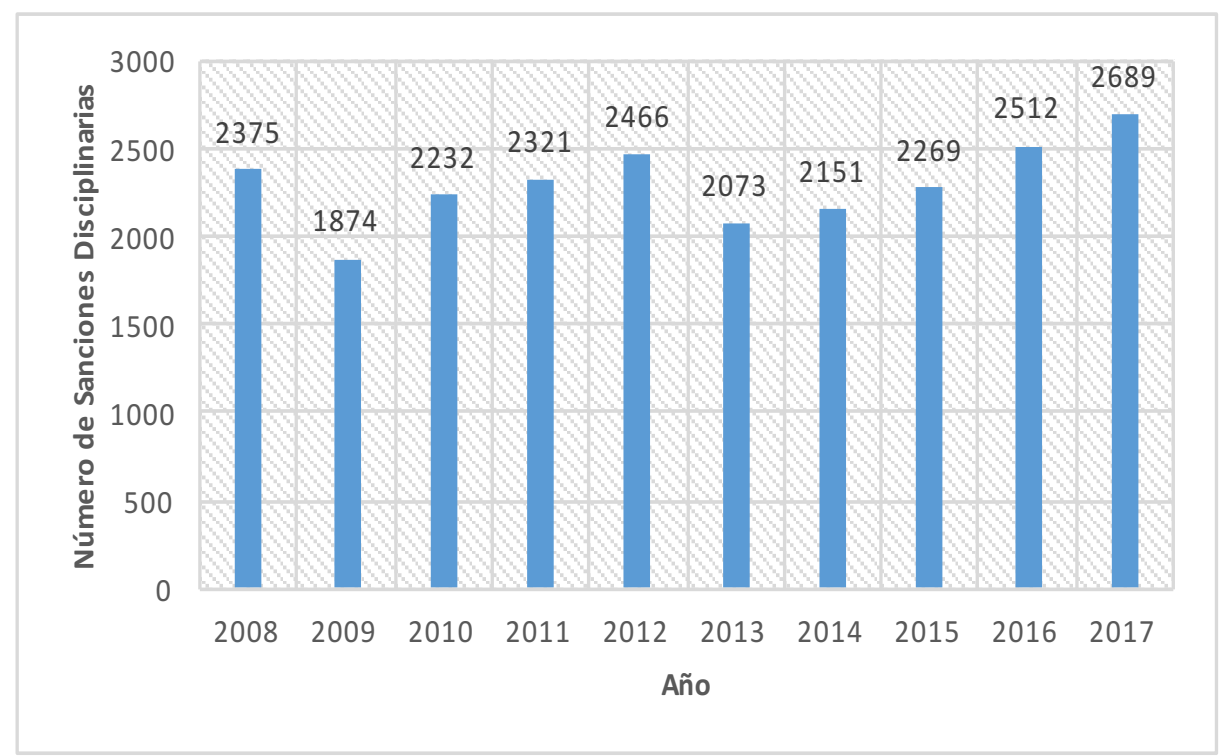

Gráfica 1. Sanciones Disciplinarias de 2008 a 2017.

Fuente: elaboración propia, con datos del Observatorio de Transparencia y Anticorrupción, 2019. (Observatorio de Transparencia y Anticorrupción, 2019)

Como se puede ver en la gráfica 1, la imposición de sanciones en el ámbito disciplinario presenta fluctuaciones importantes en el tiempo, tanto así que podemos concluir que durante el periodo de la medición, la aplicación de las normas disciplinarias no ha sido homogénea en términos de sanciones, lo cual no necesariamente puede ser malo o bueno per se, ya que se deben analizar los momentos puntuales y los contextos en que se aumenta el número de sanciones o disminuye el número de estas.

Simultáneamente distinguimos tres momentos centrales, el primero de 2008 a 2009 en donde se presentó un descenso importante, un total de 500 sanciones menos respecto al año inmediatamente anterior, hecho que resulta contradictorio si se tiene en cuenta la entrada en vigencia de la Ley 1015 de 2006 o Régimen Disciplinario de la Policía Nacional y la Ley 1123 de 2007 o Código Disciplinario del Abogado, ya que supondrían un aumento de sanciones al incluir un mayor número de conductas tipificadas, las razones corresponderían a situaciones políticas y sociales, como lo son el cambio de Procurador General de la Nación de Edgardo Maya Villazón (2001 a 2009) a Alejandro Ordoñez Maldonado (2009 a 
2016); vemos como el período 2008 a 2009, representa una etapa de tránsito de mandato y de lobby político, lo cual puede influir en esta caída de 500 sanciones disciplinarias.

El segundo momento va desde 2009 a 2012, periodo en el que se observa un aumento progresivo de las sanciones, como hecho significativo a nivel de legislación se destaca la entrada en vigencia del Estatuto Anticorrupción en el año 2011, así mismo, se ve la influencia de los tres primeros años en el cargo del Ex procurador General de la Nación Alejandro Ordoñez Maldonado, lo cierto es que de 2009 a 2012 existe un aumento de 592 sanciones. Finalmente, el tercer momento va desde el año 2013 a 2017, período durante el cual el aumento de las sanciones es progresivo en el tiempo, pasando de 2073 sanciones en 2013 a 2689 en 2017, es principalmente en este incremento sobre el cual recae nuestra atención, ya que es evidente una modificación de la estructura por medio de la cual actúan los funcionarios y las formas en como la conciencia del sujeto disciplinable incurre en la ilicitud, generalmente influenciado por variables como hechos sociales o políticos, lo cual se ve reflejado en las cifras expuestas (Gómez Pavajeau, 2018).

Por otra parte, se debe considerar que la aplicación de la norma disciplinaria es un hecho constatable a partir del aumento o disminución del número de sanciones impuestas en un período determinado, pero esas fluctuaciones del número de sanciones pueden deberse a diferentes variables, las cuales se relacionan, en primer lugar, con la entrada en vigencia de normas cada vez más severas y restrictivas de comportamientos, que generan una mayor incursión en faltas disciplinarias y posteriormente en más sanciones; un ejemplo de esto pueden ser nomas que penalicen aspectos relacionados con la moral y buenas costumbres, el decoro en las actuaciones, es decir que pueden ir más allá del cumplimientos de las funciones encomendadas (Mejía Ossman \& Mejía Acosta, 2009).

En segundo lugar, un aumento sustancial del número de servidores públicos, es decir, más personas que pueden incurrir en faltas disciplinarias, pero como consecuencia del aumento de la planta de personal y no de la norma en sí. En tercer lugar, la pérdida de la vocación preventiva del derecho disciplinario, que puede reunir a las dos razones anteriores, y significa que la naturaleza del régimen deja de ser preventiva y organizacional para convertirse en punitiva, en donde, en aras de conservar la buena marcha de la administración pública y del servicio público, se ha considerado constitucionalmente válido por parte de la Corte Constitucional disciplinar comportamientos que afectan la debida ejecución de las funciones públicas, ampliando no necesariamente en los espacios y periodos en donde se presta el servicio sino también en las acciones privadas y cotidianas de los funcionarios públicos, lo cual extiende el campo de aplicación del derecho disciplinario (Fernández, Parodi, Torregroza, Vásquez y Zetién, 2013).

\section{Normas cada vez más restrictivas y con más faltas disciplinarias \\ "En Colombia existen leyes para regular cualquier tipo de comportamiento, su producción} es en serie y pocas veces se evalúa de manera consciente que la obligación del legislador no se limita a expedir normas válidas" (Angarita Feo, 2018), esto implica que, los problemas de todo orden en mayor o en menor medida pretenden ser solucionados mediante la creación de normas, en correspondencia con una cultura legalista que no es ajena a varios de los países latinoamericanos; una de las consecuencias de buscar soluciones mediante la expedición de normas es la pérdida de los análisis profundos como la cultura o el contexto social, a partir de esto se puede explicar esa pérdida de la vocación preventiva del derecho disciplinario, un ejemplo de esto se puede ver en el siguiente escenario.

Una vez las discusiones sobre seguridad y conflicto armado pasan, no en su totalidad claro está, a segundo plano, la lucha contra la corrupción parece aflorar en el escenario político y mediático del país, hecho que ha generado que las preocupaciones en torno a cómo 
eliminar o por lo menos menguar este problema ganen un lugar en la agenda legislativa y política del Congreso de la República y del Ejecutivo de la Nación. Es por ello que, una de las soluciones plausibles es la utilización del derecho disciplinario como herramienta en la lucha anticorrupción, lo cual implicó la creación del estatuto anticorrupción, que trae consigo un mayor control en las actuaciones de los servidores públicos y en una legislación cada vez más severa y de mayor amplitud en términos de lo que está prohibido, hecho nocivo, no en cuanto a la lucha contra la corrupción, sino contra el hecho de que la naturaleza preventiva y organizacional del derecho disciplinario se ve abocado a adquirir un rol punitivo, que correspondería por regla general al derecho penal.

A partir del escenario anterior, podemos decir que, el desarrollo legislativo a primera vista tiene una relación directa con el aumento o disminución de las sanciones, esta idea aplica a las además áreas del derecho. Frente al régimen disciplinario, podemos ver la existencia de estabilidad normativamente, si se lo compara otros regímenes del Estado muestra de esto, es el último Código Único Disciplinario contenido en la Ley 734 de 2002, que tendrá una vigencia de 17 años, cuando salga del ordenamiento jurídico, así mismo, otros regímenes especiales, con excepción al de las fuerzas militares que se modificó en 2017, han gozado de estabilidad.

Para ejemplificar este hecho, en materia tributaria desde 2007 a 2017 se han realizados las siguientes reformas Ley 1111 Reforma Tributaria de 2006, Ley 1370 Reforma Tributaria de 2009, Ley 1430 Reforma Tributaria de 2010, Ley 1607 Reforma Tributaria de 2012, Ley 1739 Reforma Tributaria de 2014, Ley 1819 Reforma Tributaria de 2016 (Fuentes Marín \& Hinestroza, 2017).

Lo importante en este desarrollo normativo es la preservación de los derechos fundamentales, máxime cuando el ejercicio del poder punitivo del Estado no se hace en procura del interés general, ni con referencia de los límites estrictos al ejercicio del poder, para evitar desembocar en la arbitrariedad (Rojas López, 2009), ya que se han identificado casos de limitaciones a la vida privada o limitaciones en las actuaciones que pueda desarrollar en su fuero íntimo, con la expectativa de mejorar los procesos y salvaguardar a los principios de la administración pública.

Sobre las discusiones respecto a las limitaciones a la vida privada, o al establecimiento como faltas disciplinarias algunos hechos que no tienen relación con la prestación correcta del servicio, la Corte Constitucional ha realizado un amplio estudio, en donde se ha determinado la inconstitucionalidad de varias disposiciones, entre ellas términos ambiguos o prohibición de comportamientos que no repercuten en las labores de los funcionarios públicos, pero que se sancionan como si lo hicieran, lo cual es ejemplo de la ampliación de conductas sancionables.

Uno de los principales hallazgos de esta investigación, se encuentra en la falta de correspondencia entre el aumento de sanciones de 2013 en adelante evidente en la gráfica 1 , y la disminución del número de investigaciones disciplinarias a funcionarios que tienen cargos de dirección en las distintas entidades del Estado, esto implica que el incremento de las sanciones solo parece aplicar a funcionarios que no tienen cargos de dirección, es decir quienes no toman las decisiones trascendentales al interior de las entidades, pues como se muestra en la siguiente gráfica, el número de procesos disciplinarios ha caído en los cargos de dirección. 


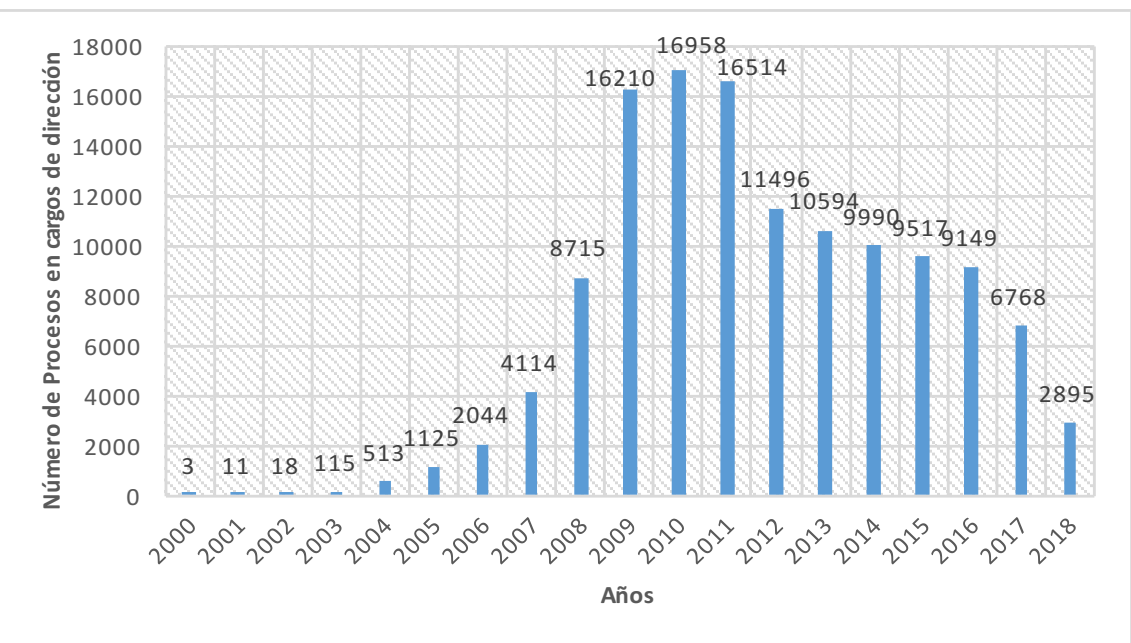

Gráfica 2. Número de investigaciones en Cargos de Dirección desde 2000 hasta 2018 Fuente: Elaboración propia con datos proporcionados por la Procuraduría General de la Nación, por medio de petición, mayo de 2019.

Como se puede evidenciar, los años con la mayor cantidad de procesos disciplinarios contra funcionarios públicos de dirección fueron 2009, 2010 y 2011, estos tres años acumularon un total del 49.682 procesos, cifra ni siquiera cercana a la sumatoria de los procesos para el mismo tipo de funcionarios desde el año 2000 hasta 2008, estos aumentos pueden ser explicados a partir de procesos derivados de escándalos sobre corrupción o prácticas ilegales desde la entidades públicas, un ejemplo de esto son las llamadas 'chuzadas' del desaparecido Departamento Administrativo de Seguridad, que tuvieron aparición mediática en febrero de 2009, así como el sonado caso Agro Ingreso Seguro, el cual terminó en la condena del exministro de agricultura de aquella época.

Fueron interceptaciones ilegales (coloquialmente referidas como 'chuzadas') del Departamento Administrativo de Seguridad -DAS- a magistrados, políticos de oposición, periodistas, entidades internacionales de Derechos Humanos y ONG (Rettberg, 2010).

El Departamento Administrativo de Seguridad -DAS - fue liquidado oficialmente durante el mandato presidencial de Juan Manuel Santos en el año 2011, luego del escándalo de las interceptaciones (Semana, 2019).

En 2010 se destapa el carrusel de la contratación en Bogotá, en este mismo año se produjeron sanciones consistentes en destituciones e inhabilidades de los gobernadores del Valle del Cauca, Casanare, Bolívar, Putumayo, Arauca, Cauca, Vichada, Guaviare y Chocó. Así también, en 2011 se desarrollaron varias coyunturas entorno a la salud, la DIAN y funcionarios de elección popular. Esto no significa, por otra parte, que todos los procesos disciplinarios de estos años sean consecuencia de los hechos coyunturales expuestos, sino que son un ejemplo de la relación entre la realidad política y el funcionamiento del aparato disciplinario.

Pero más allá de lo anterior, nuestro eje de análisis recae sobre los últimos años de 2012 a 2018, puesto que, a diferencia de las sanciones en términos generales presentados en la gráfica 1, aquí se presenta una disminución, lo cual permite concluir que las sanciones han aumentado, pero no precisamente en los cargos de dirección, sino sobre cargos que no tienen un poder amplio de decisión, esto nos lleva a preguntarnos si ¿el progresivo aumento de sanciones disciplinarias no es responsabilidad también de quienes tienen cargos de dirección? Lo que sí es claro, por otro lado, es que el aumento de sanciones y la disminución de procesos disciplinarios en cargos de administración no tienen una relación lógica que permita explicarlos. 


\section{Aumento de empleados públicos}

Como se mencionó, el aumento de las sanciones disciplinarias puede ser causado por distintas variables, y una que debe ser tenida en cuenta es el aumento del número de empleados públicos en Colombia. Frente a este tema se debe decir que la Comisión del Gasto no cuenta con una cifra cierta del tamaño del empleo público (Fasecolda, 2018), lo cual, de entrada, resulta un problema, puesto que parafraseando a William Thomson Kelvin (1824 - 1907) lo que no se mide, no se puede mejorar; esto abre la discusión en torno a un problema institucional en la medición, así como de la complejidad de medir y tener precisión de los fenómenos sociales, pero más allá de esto, según cálculos de Fasecolda en 2017, el sector público contaba con alrededor de 1.86 millones de trabajadores. Este número resulta superior en un $29 \%$ al que presenta el informe final de la Comisión del Gasto para el mismo año (Fasecolda, 2018), dicha diferencia radica en los parámetros que tiene la comisión mencionada para realizar la medición, este escenario no permite analizar el aumento de las sanciones y su relación con el crecimiento progresivo de empleados del Estado. La diferencia radica en que la Comisión del Gasto registra a los empleados que el Estado reconoce, mientras que Fasecolda el número de cotizantes que registran que tienen vinculación con el Estado (Fasecolda, 2018).

Pese a la situación anterior, queremos proponer un ejercicio que permita visibilizar la relación entre estas dos variables en un período determinado y, para ello, presentamos el número de funcionarios públicos por región y el número de sanciones disciplinarias en el 2017, como se muestra en la siguiente gráfica.

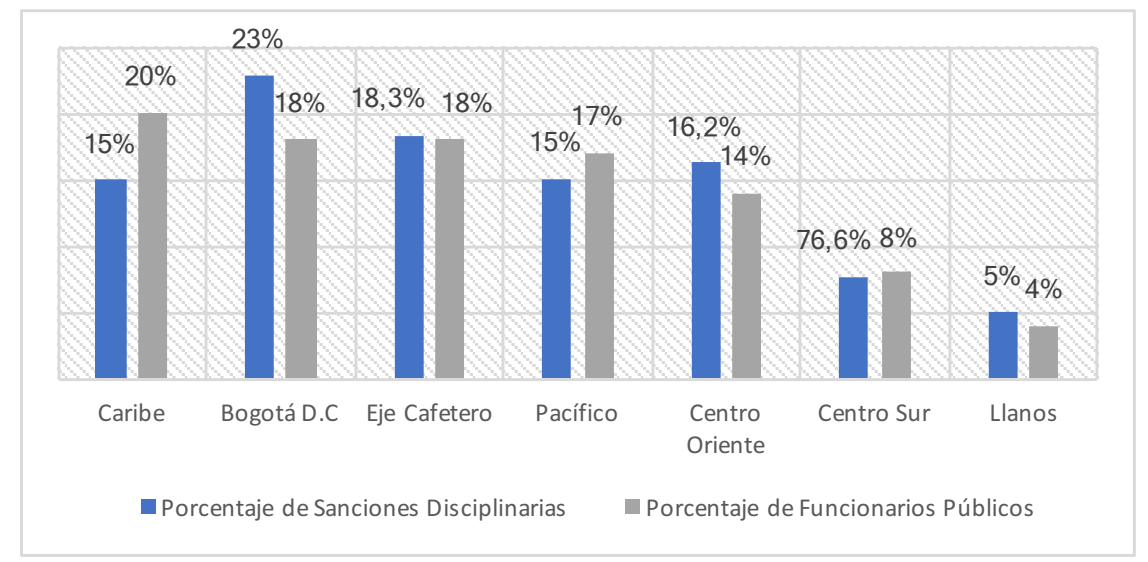

Gráfica 3. Número de funcionarios públicos y número de sanciones disciplinarias en 2017 por región. Fuente: elaboración propia con información del Observatorio de Transparencia y Anticorrupción (Observatorio de Transparencia y Anticorrupción, 2019); (Fasecolda, 2019)

Como se puede ver en la gráfica 3, es indudable la existencia de una relación entre las dos variables, sin embargo, esta no explicaría el aumento progresivo de las sanciones disciplinarias en Colombia, de ser así la región con más funcionarios públicos sería a su vez la región con más sanciones disciplinarias, y para el caso, vemos que la región Caribe concentra al $20 \%$ de los funcionarios del país y las sanciones disciplinarias solo llegan al $15 \%$ del total nacional, cifra por debajo de regiones como el Eje Cafetero con 18\% de las sanciones, región Centro Sur con 16,2\% y Bogotá D.C. con 23\%, lo cual indica que el hecho de tener más funcionarios no significa necesariamente más sanciones. 


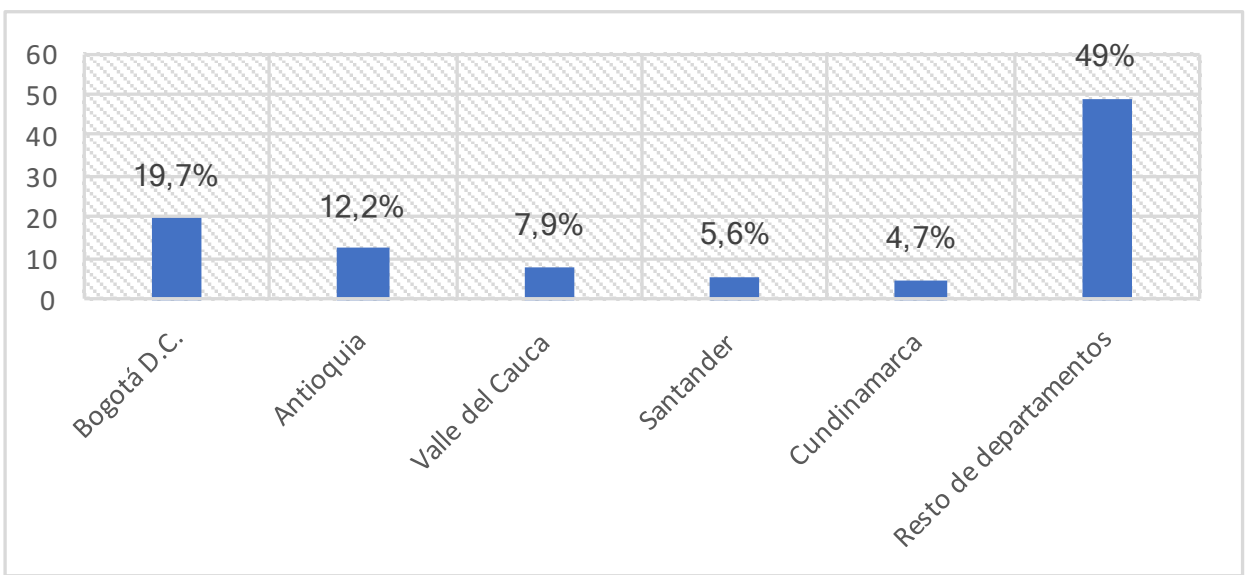

Gráfica 4, número de sanciones disciplinarias en 2017 por departamento.

Fuente: Elaboración propia con información del Observatorio de Transparencia en 2019.

(Observatorio de Transparencia y Anticorrupción, 2019)

El escenario es distinto si el análisis se realiza a nivel de departamentos, en la medida en que se puede ver una tendencia, con excepción de Atlántico con Barranquilla, en cuanto a los lugares con más funcionarios públicos y más sanciones disciplinarias, por lo menos en las tres ciudades más grandes de país. Bogotá aporta el $19.7 \%$ de las sanciones, un total de 4.290 casos, Antioquia el 12.2\% con 2.649 sanciones, Valle del Cauca el $7.9 \%$ con 1.727, después está Santander con el 5.6\% con 1.220 y Cundinamarca un 4.7\% con 1.031; en estos departamentos se han impuesto el $50.1 \%$ sanciones disciplinarias por temas de corrupción, para un total de 10.917 sanciones disciplinarias.

Es claro que un elevado número de sanciones es el reflejo de un problema en cuanto al cumplimiento de la norma, pero también "es muestra de la visión errónea de que el derecho disciplinario es para sancionar" (Roa Salguero, 2019), lo cual de plano deja en segundo lugar a la razón principal para la cual fue creado, que en últimas es que la función pública sea llevada de forma eficiente.

\section{Conclusiones}

Es claro que el aumento progresivo de las sanciones disciplinarias no son un hecho aislado o ajeno a las dinámicas sociales y políticas de Colombia, podemos decir que tanto el desarrollo normativo en términos de creación de leyes, así como las diferentes posturas jurisprudenciales respecto al tema influyen en la tendencia de crecimiento, también lo hacen las condiciones sociales y los eventos coyunturales que involucran responsabilidad de funcionarios del Estado y, específicamente, en el aumento de funcionarios públicos se puede ver la relación entre la concentración de funcionarios en un territorio y las sanciones disciplinarias que se imponen en este.

Teniendo en cuenta lo anterior, se debe aclarar que, el aumento de sanciones respalda la idea de la pérdida de vocación, es decir, que el derecho disciplinario ha enfocado sus esfuerzos en la imposición de sanciones, siendo un derecho punitivo y asimilándose cada vez más al derecho penal, en donde la función de organización y prevención se ve relegada. Creemos que es importante retomar un estudio y aplicación del derecho disciplinario más allá de la sanción, el cual se preocupe por la correcta vigilancia de los procesos, este ejercicio sin duda desembocará en una menor incursión en faltas disciplinarias, esto tiene que ver con la premisa de prevenir antes de sancionar, pues es claro que el éxito de la normatividad disciplinaria no se encuentra en el número de sanciones, por lo que el incremento de estas son muestra de un comportamiento desacertado de quienes ejercen la función pública. 
El éxito del derecho disciplinario no se puede medir a partir del número de sanciones que se impongan, ya que no son señal de eficiencia y eficacia del régimen disciplinario, los indicadores de gestión que se fundamentan en la cantidad de funcionarios destituidos o inhabilitados no son fieles a la naturaleza preventiva y organizacional con la que fue pensada el sistema disciplinario colombiano, el cual desafortunadamente se ha convertido en una herramienta política que busca sacar a contrincantes políticos de esferas públicas, es importante que el operador disciplinario no haga uso desmedido y arbitrario de la potestad disciplinaria.

La responsabilidad disciplinaria está relacionada con el ejercicio responsable de la sanción, es claro que existe una desigualdad entre quienes tienen cargos de dirección y quienes no tienen este tipo de responsabilidades, pues mientras en los primeros el número de investigaciones se ha reducido, en los segundos, los datos demuestran que existe un aumento. Esto debe ser un llamado de atención para quienes ejercen la potestad disciplinaria toda vez que la aplicación de la norma debe ser acorde a las dinámicas y de acuerdo a las responsabilidades, pero también a partir de la vocación preventiva y organizacional del derecho disciplinario.

\section{Referencias Bibliográficas}

Angarita Feo, J. E. (2018). Colombia: país donde abundan las leyes y escasea la legalidad. Razón Crítica, 195-217. doi: http//dx.doi.org/10.21789/25007807.1302

Asamblea Nacional Constituyente. (4 de Julio de 1991). Constitución Política de Colombia. Bogotá D.C., Colombia: Congreso de la República de Colombia. Recuperado de: http://www.secretariasenado.gov.co/index.php/constitucion-politica

Bernal Cuéllar, J. (1999). Exposición de motivos a la Ley 734 DE 2002. Secretaría Jurídica de la Alcaldía Mayor de Bogotá. Recuperado de: https://www.alcaldiabogota.gov. co/sisjurMantenimiento/normas/Norma1.jsp?i=11412

Congreso de la República de Colombia. (5 de febrero de 2002). Ley 734 de 2002, Por la cual se expide el Código Único Disciplinario. Diario oficial No. 44708. Recuperado de: https://www.procuraduria.gov.co/guiamp/media/file/Macroproceso\%20Disciplinario/L-734-02.htm

Congreso de la República de Colombia. (20 de agosto de 1913). Ley 4 de 1913, Sobre el régimen político y municipal. Diario oficial No. 15012. Recuperado de: http://www. secretariasenado.gov.co/senado/basedoc/ley_0004_1913.html

Congreso de la República de Colombia. (29 de octubre 1938). Ley 165. de 1938, Por lo cual se crea la carrera administrativa. Diario oficial No. 2335. Recuperado de: https:// www. funcionpublica.gov.co/eva/gestornormativo/norma. php?i=8580

Congreso de la República de Colombia. (2014). Proyecto de ley 'Código Disciplinario Único'. Gaceta del Congreso. Recuperado de: http://leyes.senado.gov.co/proyectos/images/documentos/Textos\%20Radicados/Ponencias/2014/gaceta_401.pdf

Congreso de la República de Colombia. (28 de enero de 2019). Ley 1952 de 2019. Ley por Medio de La Cual se Expide el Código General Disciplinario se Derogan la Ley 734 de 2002 y Algunas Disposiciones de la Ley 1474 de 2011, Relacionadas con el Derecho Disciplinario. Diario oficial No. 50850. Recuperado de: https://www.funcionpublica.gov.co/eva/gestornormativo/norma.php?i=90324

Corte Constitucional de Colombia. Sala plena. (22 de mayo del 2002). Sentencia C-391. Demanda de inconcstitucionalidad contra el artículo 25, numeral 10, de la Ley 200 de 1995. [MP Jaime Córdoba Triviño]. Recuperado de: http://www.corteconstitucional.gov.co/relatoria/2002/c-391-02.htm 
Corte Constitucional de Colombia. (4 de octubre de 1993). Sentencia C-417 de 1993. Demanda de incostitucionalidad contra el artículo 51 del Decreto 1888 de 1989. [MP José Gregorio Hernández Galindo]. Recuperado de: http://www.corteconstitucional.gov.co/relatoria/1993/C-417-93.htm

Corte Constitucional de Colombia. (25 junio de 1996). Sentencia C-280. [MP Alejandro Martínez caballero]. Recuperado de: http://www.corteconstitucional.gov.co/relatoria/1996/c-280-96.htm

Corte Constitucional de Colombia. (10 diciembre de 1998). Sentencia C 769. [MP Antonio Barrera Carbonell]. Recuperado de: http://www.corteconstitucional.gov.co/relatoria/1998/C-769-98.htm

Corte Constitucional de Colombia. (12 marzo de 2002). Sentencia C-181. Demanda de inconstitucionalidadcontra los artículos $9^{\circ}$ (total), 20, 25, 27, 29, 3044 (parciales), 65 (total), 116, 131, 146, 151 y 157 (parciales) de la Ley 200 de 1995. [MP Marco Gerardo Monroy Cabra] Recuperado de: http://www.corteconstitucional.gov.co/relatoria/2002/C-181-02.htm.

Fasecolda. (2018). Trabajo Formal En Colombia, Realidad y Retos. Bogotá D.C.: Federación de Aseguradores Colombianos.

Fernández Córdoba, J., Parodi Gámez, M. C., Torregroza DíazGranados, L. C., Vásquez Flórez, D. A., \& Zetién Castillo, J. A. (2013). El Injusto del Derecho Disciplinario. Revista Derecho Penal y Criminología, 34(97), 159 -174.

Fuentes Marín, J. V., \& Hinestroza, J. (2017). Estudio de las reformas tributarias en Colombia y sus efectos sobre variables macroeconómicas. Bogotá D.C: Universidad Católica de Colombia.

Gómez Pavajeau, C. A. (2018). Neuroderecho Penal Y Disciplinario. Bogotá D.C.: Universidad Externado.

Gómez Pavajeau, C. A. (2011). El derecho disciplinario en Colombia. 'Estado del arte'. Revista Derecho Penal y Criminología, 32 (92), 115-154.

Gómez Pavajeu, C. A. (2009). Problemas Centrales del Derecho Disciplinario. Bogotá D.C: Ediciones Nueva Jurídica.

Instituto de Estudios del Ministerio Público. (2007). Lecciones de Derecho Disciplinario, Vol. II. Bogotá D.C.: Procuraduría General de la Nación.

Instituto de Estudios del Ministerio Público. (2009). Lecciones de Derecho Discipliario Volumen 13. Bogotá D.C: IEMP Ediciones.

Instituto de Estudios del Ministerio Público. (2011). Código Disciplinario Único Notas de Vigencia 2011. Bogotá: IEMP Ediciones.

Isaza Serrano, C. M. (2009). Teoría General del Derecho Disciplinario. Bogotá D.C.: Temis. S.A.

Mejía Ossman, J., \& Mejía Acosta, k. (2009). Tématica Jurisprudencial del Proceso Disciplinario. Bogotá D.C.: Ediciones Doctrina y Ley LTDA.

Mora, L. (2009). Lecciones de Derecho Disciplinario. Bogotá D.C.: Instituto de Estudios Técnicos de la Procuraduría General de la Nación.

Nieto, A. (2003). Problemas Capitales del Derecho Disciplinario. Boletín Oficial del Estado, $39-84$.

Observatorio de Transparencia y Anticorrupción. (24 de abril de 2019). Indicador de sanciones disciplinarias. Recuperado de: http://www.anticorrupcion.gov.co/Paginas/ indicador-sanciones-disciplinarias.aspx

Rettberg, A. (2010). Colombia 2009, Progreso e Incertidumbre. Revista de Ciencia Política, 30(2) 249 - 273. doi: http://dx.doi.org/10.4067/s0718-090X2010000200005

Revista Semana. (10 de junio de 2019). El DAS deja de existir para dar paso a la Agencia Nacional de Inteligencia. Obtenido de https://www.semana.com/politica/articulo/ el-das-deja-existir-para-dar-paso-agencia-nacional-inteligencia/248740-3 
Roa Salguero, D. (2 de julio de 2019). El Incremento de las sanciones disciplinarias en Colombia. (H. H. Mosquera, Entrevistador)

Rojas López, J. G. (2009). El garantismo en el marco del derecho administrativo sancionado. El Ágora usb, 9 (2), 373 - 389. doi: http://doi.org/10.215000/16578031.417

Nota

Este artículo de investigación es resultado del proyecto de investigación denominado "La afectación a la vida privada del Servidor Público en Colombia de 2000 a 2017" el cual fue ganador de la convocatoria interna de financiación de proyectos en la Corporación Universitaria Autónoma del Cauca 2018. 